\title{
Construction on Index System of Martial Arts Modernization Development
}

\author{
Yu Xue 1, a \\ ${ }^{1}$ Physical Education Department Northwest University, Xi'an, Shaanxi, China \\ axueyu8528@163.com
}

Key Words: Index System; Martial Arts; Modernization Development

\begin{abstract}
Against the background of society modernization, especially in the process of constructing a harmonious society, Martial arts as a classic representative of Chinese traditional sports needs coordinated development with the modernized society. The main goal of this article is to construct an index system of martial arts modernization. The index system can reflect the condition of martial arts as a tool, and have an objective evaluation for the modernization level of Martial Arts. This study used the Delphi method. Firstly, we interviewed some experts and formulated the preliminary questionnaire of martial arts modernization according to the constructive principles and the characteristics of martial arts. Secondly, we used the Delphi method on two rounds of anonymous expert investigation to establish the evaluation index system of martial arts modernization. We chose 15 experts in the field of martial art or other related. After the first round of expert investigation, we revised our questionnaire, sent the new questionnaire to the experts and collected them. Lastly, we did data analysis via SPSS 16.0.The results of this paper identified eight primary class indexes and 21 secondary indexes as a martial arts modernization evaluation index system. And the mean of the selected indexes was above 3.5; variation coefficients (CV) were less than 0.25; each group's coordinate coefficient (W) indicator was above 0.4 ; and the consistency of coordination degree test was $\mathrm{P}<0.01$. It is suggested that the experts considered the result consistency of each index evaluation was higher. The coordination degree was good. So the results were reliable. This study also established the weights of each layer of the index system of martial arts modernization. And this index system will have extended and well applicable prospects.
\end{abstract}

\section{Methods}

This study used the Delphi method. Firstly, we interviewed some experts and formulated the preliminary questionnaire of martial arts modernization according to the constructive principles and the characteristics of martial arts. Secondly, we used the Delphi method on two rounds of anonymous expert investigation to establish the evaluation index system of martial arts modernization. We chose 15 experts in the field of martial art or other related. After the first round of expert investigation, we revised our questionnaire, sent the new questionnaire to the experts and collected them.

\section{Data Analysis}

This paper did data analysis via SPSS 16.0. We got the date about the mean (M), variation coefficients (CV) of every index, and the coordinate coefficient indicator (W) of every group. At the same time, we analyzed these date's meaning and test the consistency of coordination degree. 


\section{Results}

The results of this paper identified eight primary class indexes and 21 secondary indexes as a martial arts modernization evaluation index system.

Table1 Descriptive Data of Primary Class Indexes

\begin{tabular}{lccc}
\hline Primary Class Indexes & Mean & Std. & CV \\
\hline Mass martial arts & 5 & 0 & 0 \\
School martial arts & 5 & 0 & 0 \\
Competition martial arts & 4.3 & 0.483 & 0.11 \\
Martial arts industry & 4 & 0.667 & 0.17 \\
Martial arts management & 4.6 & 0.516 & 0.11 \\
Martial arts information & 4.5 & 0.850 & 0.19 \\
Martial arts culture & 4.3 & 0.823 & 0.19 \\
Martial arts technology & 3.8 & 0.422 & 0.11 \\
\hline
\end{tabular}

From the table1, the mean of the selected indexes was above 3.5; variation coefficients (CV) were less than 0.25; these date means all the experts considered the indexes is good and reasonable.

Table2 The Consistency of Coordination Degree Test the Primary Class Indexes

\begin{tabular}{cccc}
\hline Round & $\mathrm{N}$ & Kendall's W & Asymp.Sig (P) \\
\hline Second round & 10 & .441 & .000 \\
\hline
\end{tabular}

From table2, this group's coordinate coefficient (W) indicator was above 0.4; and the consistency of coordination degree test was $\mathrm{P}<0.01$. It is suggested that the experts considered the result consistency of each index evaluation was higher. The coordination degree was good. So the results were reliable. This study also established the weights of each layer of the index system of martial arts modernization. And this index system will have extended and well applicable prospects.

Table3 Descriptive Data of the Secondary Class Indexes

\begin{tabular}{lccc}
\hline Secondary Class Indexes & Mean & Std. & CV \\
\hline Safeguarding conditions & 4.7 & 0.483 & 0.10 \\
Martial arts degree of organization & 5 & 0 & 0 \\
Martial arts teachers & 5 & 0 & 0 \\
Classes and activities & 4.7 & 0.483 & 0.10 \\
Funds facilities & 4.1 & 0.316 & 0.08 \\
Martial arts competition & 4.7 & 0.483 & 0.10 \\
Martial arts training & 5 & 0 & 0 \\
Martial arts value & 4.4 & 0.516 & 0.12 \\
Martial arts consumption & 4 & 0.667 & 0.17 \\
The industrial structure & 4 & 0.667 & 0.17 \\
Human resources & 4.6 & 0.516 & 0.11 \\
Management system & 4.9 & 0.316 & 0.06 \\
Management team & 4.3 & 0.483 & 0.11 \\
Management ways & 4.8 & 0.422 & 0.09 \\
Spreading basic conditions & 5 & 0 & 0 \\
\hline
\end{tabular}




\begin{tabular}{lccc}
\hline Communication effect & 5 & 0 & 0 \\
Martial arts culture consciousness & 4.5 & 0.527 & 0.12 \\
Wushu cultural behavior & 4.5 & 0.527 & 0.12 \\
Scientific research team & 4.7 & 0.483 & 0.10 \\
Technology guarantee & 4.6 & 0.516 & 0.11 \\
Science and technology achievements & 4.5 & 0.527 & 0.12 \\
\hline
\end{tabular}

The mean of the secondary class indexes was above 3.5; variation coefficients (CV) were less than 0.25 ; these date means all the experts considered the indexes is good and reasonable.

Table4 The Consistency of Coordination Degree Test of the Secondary Class Indexes

\begin{tabular}{cccc}
\hline Round & $\mathrm{N}$ & Kendall's W & Asymp.Sig (P) \\
\hline Second round & 10 & .504 & .000 \\
\hline
\end{tabular}

The coordinate coefficient (W) indicator of the secondary class indexes was above 0.4 ; and the consistency of coordination degree test was $\mathrm{P}<0.01$. It is implied that the experts considered the result consistency of each index evaluation was higher. The coordination degree was good. So the results were reliable. This study also established the weights of each layer of the index system of martial arts modernization. And this index system will have extended and well applicable prospects.

\section{Discussion}

The primary aim of this research was to construct an index system of martial arts modernization. The index system can reflect the condition of martial arts as a tool, and have an objective evaluation for the modernization level of Martial Arts.

In the present study, the research about the index system of martial arts development had constructed four primary index and 10 secondary indexes. Many researchers focused on the topic of martial arts modernization nowadays. But the research about how to construct the index system of martial arts modernization was very few and not all-inclusive. One limitation of the present work was that every study had no data analysis.

This study shows that each group's coordinate coefficient (W) indicator was above 0.4 ; and the consistency of coordination degree test was $\mathrm{P}<0.01$.This index system was good.

It is also shows that the modernization level of Martial arts can be measured by the indexes. Choose some indexes which can reflect the martial arts statement as the tool, cannot only reflect the speed of martial arts modernization development, but also can compare the modernization level of Chinese martial arts and the foreign traditional sport. It can find the weak link of martial arts modernization process. Hence, the purpose of this paper is to construct a set of index system which can measure the modernization development level of Martial arts. In order to have an objective evaluation for the modernization level, and provide certain theoretical basis of Martial arts modernization.

\section{Conclusion}

From this paper, we found that: (1) the modernization of martial arts is not only the modernization of itself, but also all the related aspects of martial arts. (2) The main content of martial arts modernization mainly includes mass martial arts, school martial arts, competition martial arts, martial arts industry, martial arts management, martial arts culture, martial arts information, and martial arts technology. (3)The modernization level of Martial arts can be measured by the indexes. We can use this index system to evaluate the level of modernization of martial arts and find the weak link of martial arts modernization 
process.

\section{References}

[1] He, C.Q. (Ed.). (1999) .The second modern - human civilization progress enlightenment. Beijing: Higher Education Press.

[2] Wang, G., Guo H.Z. (2007) .Martial arts modernization quota system construction. Xi'an Sports Institute Journal, 4, 1-6.

[3] Xiong, D.Y.(1980) Modern sports and sports modernization problem discussed . Beijing Sports University Journal, 1, 9 